usually be developing, emerging in October, the wild individuals oriented their flight paths to the south, and most were in reproductive diapause. But the commercially bred monarchs reared under exactly the same conditions did not exhibit directional flight (Fig. 1b) and produced as many eggs as the summer butterflies.

Genetic analyses of the commercially bred monarchs showed that they were distinct from any other wild population tested. This finding provides a crucial lesson about the fragility of the behavioural and morphological characteristics that lead monarchs that emerge in late summer or autumn to put off reproduction for many months and migrate, and monarchs that emerge earlier to reproduce just days after emerging as adults without exhibiting directional flight. How many generations of captive breeding led to the changes that resulted in the loss of migratory abilities is unknown. Regardless of the uncertainty about this, Tenger-Trolander and colleagues' study is a necessary reminder that such changes can happen.

The second conclusion of Tenger-Trolander and colleagues' study is that even wild monarchs reared in captive conditions can lose their propensity to migrate. In a separate experiment, wild North American monarchs were reared outdoors or indoors. Indoor-bred monarchs were kept in incubators in which they experienced either $25^{\circ} \mathrm{C}$ and a 16 -hour day, or $18^{\circ} \mathrm{C}$ with a 14-hour day - temperatures and day lengths that Tenger-Trolander and colleagues described as representing summer- or autumnlike conditions, respectively. The butterflies reared outside during the summer showed no directional flight, whereas those reared outside in the autumn did, as expected. But none of the butterflies reared inside in either of the incubators showed directional flight. This was even true when the monarchs were inside only for their final three days of development.

This is a sobering finding about the importance of the conditions that monarchs experience during captive rearing. However, it is not surprising that the conditions under which these monarchs were reared did not lead to migratory behaviour or diapause. The monarchs were not exposed to natural autumnal light or temperature fluctuations; instead, they experienced 14 hours of light followed by 10 hours of dark throughout the experiment, and the temperature was kept constant. These are not conditions that truly mimic autumn, when day length is changing rapidly and there are usually substantial differences between day- and night-time temperatures. Shortening days and day-night temperature fluctuations are both drivers of diapause induction ${ }^{5}$. In most small-scale inside-rearing conditions, such as in people's houses and classrooms, windows and daily temperature fluctuations are likely to provide sufficient exposure to such natural environmental cues.

Some people release monarchs that they have either purchased from commercial breeders or reared from eggs or larvae collected from the wild, with the aim of giving this butterfly population a boost. However, given the magnitude of the number of extra monarch butterflies that would be needed for these butterfly populations to reach sustainable levels ${ }^{2,6}$, there is widespread agreement that the best way to boost monarch-butterfly conservation is to protect and create the habitats that they need ${ }^{6}$. Focusing on habitat has the added benefit of also helping many other plant and animal species.

Tenger-Trolander and colleagues provide evidence that mass rearing monarchs over many generations might not only have few positive benefits, especially if the released butterflies do not migrate, but could also have negative consequences if such butterflies spread versions of genes that could thwart migration processes if introduced into wild populations. The authors used monarch butterflies from one commercial source. Many commercial breeders of monarch butterflies claim to regularly interbreed their stock with wild butterflies, which might alleviate such problems, but this industry is mainly unregulated. The results reported by Tenger-Trolander et al. confirm concerns, voiced previously by many scientists, about the consequences of the captive mass rearing of monarch butterflies.

As Tenger-Trolander and colleagues mention, rearing monarchs under suitable conditions has educational, inspirational and scientific benefits ${ }^{7,8}$. However, their recommendation that these butterflies should be reared outdoors is often not practical. The lack of exposure to decreases in day length and to fluctuating temperatures in the authors' experiments precludes drawing the conclusion that monarchs collected from the wild and reared on kitchen tables or in classrooms will not migrate.

Karen S. Oberhauser is at the University of Wisconsin-Madison Arboretum and in the Department of Entomology, University of Wisconsin-Madison, Madison, Wisconsin 53711, USA.

e-mail:koberhauser@wisc.edu

1. Tenger-Trolander, A., Lu, W., Noyes, M. \& Kronforst, M. R. Proc. Natl Acad. Sci. USA 116 14671-14676 (2019).

2. Semmens, B. X. et al. Sci. Rep. 6, 23265 (2016).

3. Thogmartin, W. E. et al. R. Soc. Open Sci. 4, 170760 (2017).

4. Herman, W. S. \& Tatar, M. Proc. R. Soc. Lond. B 268 2509-2514 (2001)

5. Goehring, L. \& Oberhauser, K. S. Ecol. Entomol. 27, 674-685 (2002)

6. Thogmartin, W. E. et al. Environ. Res. Lett. 12, 074005 (2017).

7. Oberhauser, K. S., Nail, K. R. \& Altizer, S. (eds) Monarchs in a Changing World: Biology and Conservation of an Iconic Butterfly (Cornell Univ. Press, 2015).

8. Young-Isebrand, E. et al. in Monarchs in a Changing World: Biology and Conservation of an Iconic Butterfly (eds Oberhauser, K. S., Nail, K. R. \& Altizer, S.) Ch. 1 (Cornell Univ. Press, 2015).

This article was published online on 9 September 2019.

\title{
The structure of a T-cell mechanosensor
}

T-cell receptors orchestrate immune-system responses against infection and cancer. A structure of an entire T-cell receptor complex clarifies its assembly and signalling, and sheds light on its dynamic ligand recognition. SEE ARTICLE P.546

\section{ELLIS L. REINHERZ}

$\int \mathrm{r}$ mmune cells called T cells have T-cell receptors (TCRs) on their cell membrane that recognize dysfunctional cells expressing abnormal protein fragments. Such abnormalities can arise in cells if, for example, cancer develops or infection occurs. When TCRs recognize these unusual peptides, the receptors become activated and stimulate T cells to destroy or inhibit the abnormal cells. Such T-cell responses are being harnessed for anticancer clinical therapies. TCRs are also of interest because their dysfunction can lead to autoimmunity or immunodeficiency diseases.

On page 546, Dong et al. ${ }^{1}$ present the structure of a human TCR, at a resolution of 3.7 ångströms, obtained using an imaging technique called single-particle cryogenic electron microscopy (cryoEM). Such a highresolution structure of the entire TCR was previously lacking, and it provides a wealth of detail about this receptor.

For more than 35 years $^{2}$, it has been known that each TCR of the type called an a $\beta$ TCR is a protein complex. Eight proteins form the TCR: six of these are collectively known as CD3, which acts in a signalling capacity when a TCR is activated. CD3 comprises a heterodimer of CD $3 \varepsilon$ and $\mathrm{CD} 3 \delta(\mathrm{CD} 3 \varepsilon \delta)$, a heterodimer of CD $3 \varepsilon$ and $C D 3 \gamma(C D 3 \varepsilon \gamma)$ and a homodimer of $\mathrm{CD} 3 \zeta(\mathrm{CD} 3 \zeta \zeta)$. The other two proteins that form the TCR are TCR $\alpha$ and $\operatorname{TCR} \beta$. They create the ligand-binding heterodimer (TCR $\alpha \beta)$ that recognizes a peptide bound to a major histocompatibility complex 
$\sim$ molecule (MHC) on the surface of another cell - a combination called a pMHC. TCRa, $\mathrm{TCR} \beta, \mathrm{CD} 3 \delta, \mathrm{CD} 3 \varepsilon$ and $\mathrm{CD} 3 \gamma$ contain extracellular regions (called ectodomains), $\vec{w}$ connecting-peptide regions, transmembrane regions and cytoplasmic tails.

Each person's many T cells have the same set of CD 3 proteins, but TCR $\alpha$ and TCR $\beta$ have what are called variable domains that differ between $\mathrm{T}$ cells such that different $\mathrm{T}$ cells have a unique capacity to recognize specific peptides - called antigens - in pMHCs. Much is already known $\mathrm{n}^{3-5}$ about the structure and function of individual TCR components, but an understanding of the complete TCR structure promises to provide fresh insights.

Dong and colleagues co-expressed all the TCR proteins in cultured cells, where they assembled into the TCR complex. The authors isolated the complex and stabilized it for cryoEM by chemical cross-linking, to form permanent bonds between adjacent proteins. This approach enabled the authors to obtain structural data. Given the limited interaction surface between the various CD3 dimers and the TCR $\alpha \beta$ ectodomains shown by Dong and colleagues, it is possible that alternative TCR conformations exist. The cross-linking used to obtain cryoEM data imposes constraints on structural variability.

The paired variable domains formed by the TCR $\alpha \beta$ heterodimer are located at the centre of the TCR, in the extracellular region farthest from the cell membrane (Fig. 1). The variable domains' surface for binding a pMHC is oriented in a way that is consistent with the emerging idea that the TCR acts in a directionselective manner as a mechanosensor during antigen recognition ${ }^{6}$. It binds its ligand in one direction only, owing to a physical property termed anisotropy.

Another striking structural feature of the TCR is its amalgam of the ectodomains, which consist of what are termed the constant domains of TCR $\alpha$ and TCR $\beta$, as well as the ectodomains of CD $3 \varepsilon \gamma$ and $C D 3 \varepsilon \delta$, plus the short extracellular segments of $\mathrm{CD} 3 \zeta \zeta$. Connecting peptides link ectodomains and the bundle of eight transmembrane helices, one helix for each protein of the TCR. In Dong et al.'s structure, the transmembrane helices are mainly in a parallel orientation among the digitonin detergent that is used to replace cellmembrane lipid for protein solubilization and imaging. The authors could not obtain structural information for the cytoplasmic tails of TCR proteins, which include CD3 regions that are vital for signalling during T-cell activation. Presumably, this was because such regions have high conformational flexibility in the absence of normal cell-membrane lipids.

Dong and colleagues' structure is compelling for many reasons. The authors docked structures of TCR components obtained previously using X-ray crystallography onto their structure. The locations of the ectodomains of $\mathrm{CD} 3 \varepsilon \delta$ and $\mathrm{CD} 3 \varepsilon \gamma$ relative to those of TCR $\alpha$

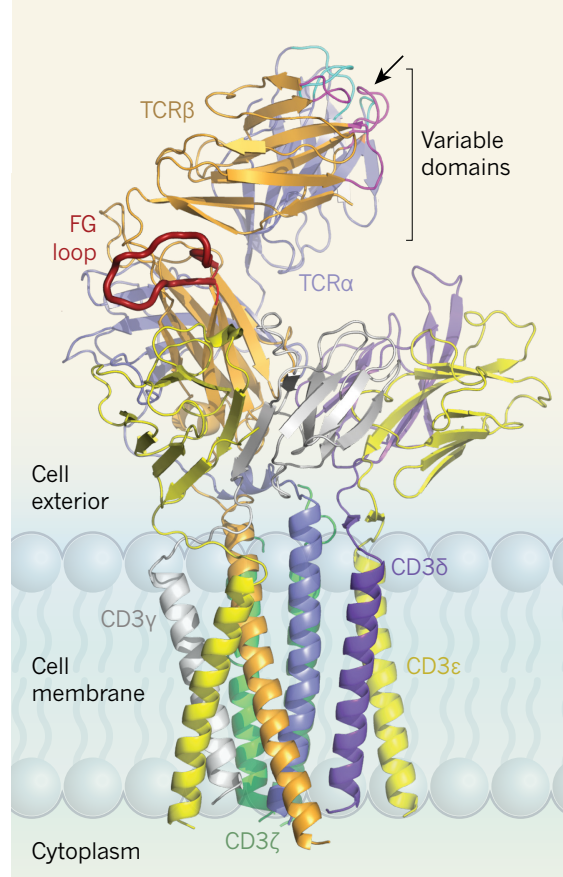

Figure 1 | The T-cell receptor. Dong et al. ${ }^{1}$ report a structure of the human T-cell receptor (TCR). This receptor orchestrates immune-defence responses when it recognizes abnormal peptides. The TCR contains eight proteins: one TCR $\alpha$ (light purple), one TCR $\beta$ (orange), two CD3 $\varepsilon$ (yellow), one CD $3 \gamma$ (white), one $\mathrm{CD} 3 \delta$ (dark purple) and two $\mathrm{CD} 3 \zeta$ (green). TCR $\alpha$ and TCR $\beta$ each have a variable domain with binding loops (cyan and magenta, respectively) that recognize a complex of a peptide bound to a protein called a major histocompatibility complex molecule (not shown), termed pMHC. If a TCR binds a pMHC that it recognizes, force transmission in such an interaction tunes its sensitivity and specificity, and the TCR therefore acts as a mechanosensor ${ }^{6}$. The TCR binds pMHC in a direction-selective manner at the site indicated by the arrow. The FG loop (red) of TCR $\beta$ 's constant region probably transmits force from the TCR's extracellular region through the membrane and onwards towards the $\mathrm{T}$ cell's interior to trigger T-cell activation and a defence response. The authors did not obtain structural data for the cytoplasmic tails of the proteins in the TCR.

and TCR $\beta$ are consistent with previous data ${ }^{5}$. Moreover, in the transmembrane region, the close juxtaposition of basic amino-acid residues in the TCR $\alpha \beta$ heterodimer and the acidic amino acids in the CD3 proteins support an earlier proposal that such interactions might help to orient the transmembrane helices ${ }^{7}$.

An evolutionarily conserved protein motif called $\mathrm{CxxC}$, found in connecting-peptide regions of $C D 3 \varepsilon \gamma$ and $C D 3 \varepsilon \delta$, has a crucial role in CD3 signalling ${ }^{8}$. Dong and colleagues report molecular connections called disulfide bridges in each of these four proteins' CxxC linkages, and also describe the protein architecture that surrounds these key linkages.

Dynamic movement of TCR $\alpha$ and TCR $\beta$ protein segments has previously been visualized using a method called $\mathrm{NMR}^{9}$. The model of the TCR as a directional mechanosensor proposes that forces exerted by T-cell motility and the motion of components of a T cell's internal framework (the cytoskeleton) improve a T-cell's recognition of pMHC more than 1,000fold relative to recognition in the absence of force ${ }^{6}$. Consequently, when a TCR interacts with a pMHC that it recognizes, a physical load is placed on the bonding between the TCR and the pMHC, paradoxically resulting in an increase in bond lifetime ${ }^{6,10,11}$, and causing dynamic structural changes in the TCR $\alpha \beta$ heterodimer that enhance the specificity and sensitivity of antigen recognition. The recognition of just one or two abnormal pMHCs among the roughly 100,000 normal pMHCs on a single cell suffices to trigger T-cell activation $^{6}$. By contrast, the interactions between a typical receptor and ligand do not rely on biomechanical forces as a crucial recognition feature. Dong and colleagues' structure provides insights into how the TCR is configured as a mechanosensor to meet its specificity and sensitivity requirements.

The orientation of the variable domains and the positioning of the antigen-binding site favours a directional interaction of the TCR with a pMHC that is consistent with T-cell scanning motions that occur tangentially to the surface of the surveyed cell. The FG loop structure ${ }^{5}$ of TCR $\beta$ 's constant region controls the lifetime of a TCR-pMHC bond and also the extension of the TCR $\alpha \beta$ heterodimer when it transitions from a compact to an extended form under force during antigen recognition $^{6,9}$. The FG loop is located above CD $3 \varepsilon$ of CD $3 \varepsilon \gamma$. If the TCR interacted with a pMHC on the right in Fig. 1, the resulting force would probably transfer, through a geometry like a lever and fulcrum, from the TCR $\alpha \beta$ heterodimer's variable domains downwards towards its constant regions and then to CD3 dimers on the same vertical plane below. The force would probably be amplified through reversible and repetitive transitioning of the TCR $\alpha \beta$ heterodimer between its expanded and contracted forms, which rearranges the TCR components.

The CxxC motifs are located near the cell membrane, and the rigid connectivity of these regions on CD3 ectodomains and the arrowhead-shaped configuration of their respective heterodimer's transmembrane segments suggests that pushing and pulling motions from TCR $\alpha \beta$ to $C D 3 \varepsilon \gamma$ or $C D 3 \varepsilon \delta$, or to both, might transfer force through the membrane or deliver this energy as work. Such forces might cause changes to parts of the TCR structure, and affect neighbouring lipids to expose key amino-acid sites on CD3 cytoplasmic tails. The addition of phosphate groups to these tyrosine amino-acid residues would trigger signalling cascades and other cellular changes needed for T-cell activa$\operatorname{tion}^{6,9,12}$. The abundance of interactions between the various connecting-peptide segments of the TCR would probably aid signalling ${ }^{1,8,13}$. 


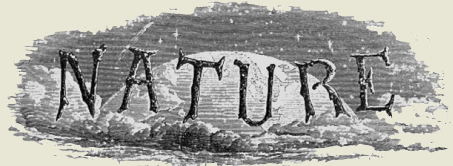

50 Years Ago

The Liberal conference which took place last week afforded the now familiar sight of the Young Liberals adopting a more radical and militant approach than that of the parent body, and being subsequently chastised for it. But the motion which had the greatest bearing on science and technology, on European unity, was largely their work. This motion commits the Liberal Party to press not only for entry into the EEC but also for a "United States of Europe" with common economic, technological and foreign policies. The Liberals see this as the only effective way to combat the increasing American dominance of European technology and consumer markets ... The motion was carried by an overwhelming majority, and this makes the Liberal Party the foremost advocate of joining the Common Market among the political parties.

From Nature 27 September 1969

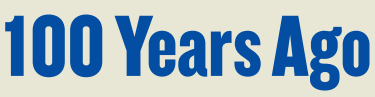

Dr. Victor E. Shelford writes in the Scientific Monthly... on the general question of the waste involved in the discharge of domestic and industrial sewage into the sea and rivers.

Experimental methods for testing the effect on fishes of various substances in solution have been devised ... The sensibility of fish to such compounds as occur in waste material is thus shown to be greater than has hitherto been supposed, thus an increase in carbon dioxide of 2 c.c. in one litre above the normal content caused the turning-away reaction. A low oxygen content was also detrimental, and this was usually found to accompany a high carbon dioxide content. The waste substances resulting from gas-production works and from munitions processes were also studied, and it was shown that these substances ... had very marked effects on fish-life.

From Nature 25 September 1919
When the authors superimposed an available structure of a pMHC bound to a TCR $\alpha \beta$ heterodimer onto their TCR structure, the TCR $\alpha \beta$ heterodimers were similar in both structures. This is unsurprising, because force application is probably the major cause of structural changes driving TCR subunit rearrangements, and these structures were obtained in the absence of force, and thus capture a compact state of the TCR $\alpha \beta$ heterodimer. The force-based TCRpMHC recognition process differs from typical receptor-ligand interactions such as antibody-antigen interactions, which are force-independent. Harnessing energy for mechanosensing from cellular motions could explain how, unlike in force-independent interactions, TCRs can discriminate so sensitively between very similar antigens, differing by just one amino acid.

It has been suggested that the subunit rearrangements that occur when force is applied to the TCR might foster CD3 dimer dissociation, starting with $\mathrm{CD} 3 \zeta \zeta$, and that this contributes to T-cell activation ${ }^{8}$. The authors' structure confirms that $\mathrm{CD} 3 \zeta \zeta$ dissociation would indeed cause changes to the TCR structure in the transmembrane region.

Dong and colleagues' work provides a basis for future studies. Could structures of other $\alpha \beta$-type TCRs of defined antigen specificities, with or without the relevant pMHC, be obtained? Might it be possible to obtain high-resolution structures of the transmembrane segments of a TCR in a natural lipid-membrane environment to visualize the cytoplasmic tails of TCR proteins? Could conformations of the TCR complex under the application of force be imaged if new structural-analysis methods are developed?

Given the importance of the TCR for understanding immune-cell function and the use of $\mathrm{T}$ cells in immunotherapy to tackle cancer, information about TCR structure might bring improvements in TCR design for medical purposes. Dong and colleagues' work is an urgent summons to immunologists interested in tumour biology and to others to consider bioforces when assessing $\mathrm{T}$ cells in vitro to gauge the potential of their TCRs in vivo. Great opportunities lie ahead to make more progress in developing high-quality TCRs for clinical use.

Ellis L. Reinherz is in the Department of Medicine, Harvard Medical School, Boston, Massachusetts 02115, USA, and at the Dana-

Farber Cancer Institute, Boston, USA.

e-mail:ellis_reinherz@dfci.harvard.edu

1. Dong, D. et al. Nature 573, 546-552 (2019).

2. Meuer, S. C. et al. Nature. 303, 808-810 (1983).

3. Davis, M. M. \& Bjorkman, P. J. Nature 334 , 395-402 (1988).

4. Rudolph, M. G., Stanfield, R. L. \& Wilson, I. A. Annu. Rev. Immunol. 24, 419-466 (2006).

5. Wang, J. H. \& Reinherz, E. L. Immunol. Rev. 250 , 102-119 (2012)

6. Feng, Y., Reinherz, E. L. \& Lang, M. J. Trends Immunol. 39, 596-560 (2018)

7. Call, M. E., Pyrdol, J., Wiedmann, M. \& Wucherpfennig, K. W. Cell 111, 967-979 (2002)

8. Brazin, K. N. et al. Immunity 49, 829-841 (2018).

9. Mallis, R. J. et al. J. Biomol. NMR 73, 319-332 (2019).

10.Liu, B., Chen, W., Evavold, B. D. \& Zhu, C. Cell 157, 357-368 (2014).

11.Sibener, L. V. et al. Cell 174, 672-687 (2018).

12. Chakraborty A. K. \& Weiss, A. Nature Immunol. 15, 798-807 (2014).

13. Bäckström, B. T. et al. Immunity 5, 437-447 (1996)

This article was published online on 9 September 2019.

\section{X-rays glimpse solid hydrogen's structure}

Little was known about the properties of hydrogen under extreme pressure. Experiments now reveal key details about the arrangement of molecules in several of the element's high-pressure phases. SEE LETTER P.558

\section{BARTOMEU MONSERRAT \\ \& CHRIS J. PICKARD}

$\mathrm{H}$ ydrogen is the most abundant element in the Universe. Our knowledge of celestial bodies such as the Sun, which is about $75 \%$ hydrogen $^{1}$, relies on understanding the properties of this element at extreme temperature and pressure. Replicating these conditions in the laboratory is exceptionally challenging, and even the structure of high-pressure phases of hydrogen at low temperatures has been an open question.
On page 558, Ji et al. ${ }^{2}$ report experiments that probe this structure at unprecedented pressures, revealing a hexagonal close-packed arrangement of molecules.

The simplicity of the hydrogen atom, which comprises a single proton and a single electron, does not prevent the high-pressure phases of the element from being rich and complex. Hydrogen is an electrical insulator at ambient conditions, but becomes a metal under extreme compression ${ }^{3}$ - a state that could, for example, help to generate Jupiter's magnetic field. Additionally, theoretical work suggests 